\title{
Chronic granulomatous disease presenting as immune thrombocytopenic purpura
}

\author{
Sumantra Sarkar ${ }^{1}$, Rakesh Mondal ${ }^{2}$, Madhumita Nandi ${ }^{1}$ \\ Sri Lanka Journal of Child Health, 2014; 43: 177-179
}

(Key words: Immune thrombocytopenia; chronic granulomatous disease)

\section{Introduction}

Immune thrombocytopenia (ITP) may co-exist with primary immunodeficiency disorders like common variable immunodeficiency (CVID), IgA and complement (C4) deficiency and Wiskott-Aldrich syndrome $^{1}$. ITP in association with chronic granulomatous disease (CGD) is rare ${ }^{2-4}$. We report a case of a 5 year old boy who presented with features of ITP and was later diagnosed as CGD.

\section{Case report}

A 5 year-old-boy, the only child of nonconsanguineous parents, presented with epistaxis, gum bleeding and generalised purpura for three days. His perinatal history and developmental milestones were normal. He had a history of repeated respiratory infections, treated by local doctors. Examination revealed generalised purpuric spots with a $6 \mathrm{~cm}$ liver and $3 \mathrm{~cm}$ spleen, other systems being normal.

His haemoglobin was $11.3 \mathrm{~g} / \mathrm{dl}$. Total and differential leucocyte counts were normal. Platelet count was $27,000 / \mathrm{cu} \mathrm{mm}$ with normal platelet morphology. Serum urea, creatinine, electrolytes, liver function, prothrombin time and activated partial thromboplastin time were normal. Abdominal sonography showed hepatosplenomegaly. Bone marrow examination revealed hypercellularity with increased myelocytes having normal maturation and increased megakaryoblasts. Erythrocytic series was normal. No abnormal cells were found. He was diagnosed as immune thrombocytopenic purpura. His platelet count rose to $84,000 / \mathrm{cu} \mathrm{mm}$ spontaneously by day 8 and he was discharged for follow up.

Four weeks later, he presented with another episode of generalised purpura with high grade fever,

${ }_{1}^{1}$ Associate Professor, Paediatric Medicine, IPGME $\& R$, Kolkata, India, ${ }^{2}$ Associate Professor Paediatric Medicine, NBMCH, Darjeeling, West Bengal, India

(Received on 5 July 2013: Accepted after revision on 30 August 2013) respiratory distress and a history of one episode of generalised tonic clonic convulsions. He had neck stiffness with positive Kernig and Brudzinski signs. Plantars were equivocal. Haemogram was unremarkable except for a low platelet count of $13,000 / \mathrm{cu} \mathrm{mm}$. CT scan, done to rule out intracranial haemorrhage, revealed multiple granulomas in left parietal, right parieto-occipital and left occipital lobes. A few granulomas at right caudate nucleus compressed the 4th ventricle causing mild hydrocephalic change. Fundoscopy demonstrated a granuloma in the right optic nerve. CSF study revealed 20 lymphocytes in high power field along with sugar $44 \mathrm{mg} / \mathrm{dl}$ and protein $240 \mathrm{gm} / \mathrm{dl}$. No acid fast bacilli were found. MRI brain demonstrated granulomas in the same areas as in CT scan with patchy areas of haemorrhagic infarction (Figure 1).

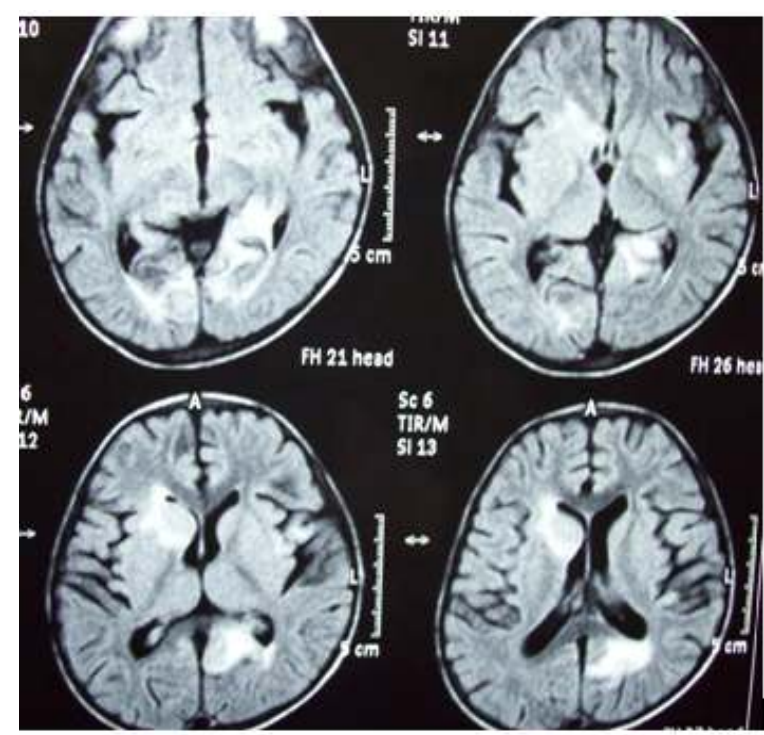

Figure 1: MRI brain showing hyper intense granulomatous lesions in right caudate nucleus and left occipital region

While investigating for high fever, fungal hyphae were detected in urine specimen that grew candida parapsilosis on fungal culture. Blood for fungal culture showed growth of aspergillus species. Chest 
X-ray revealed bilateral fluffy opacities. Tuberculin test and gastric aspirate for AFB were negative. Amphotericin B, vancomycin and ceftriaxone were started. His respiratory and neurological symptoms improved gradually and he became afebrile after 7 days. His platelet count remained low in the range of $40,000 / \mathrm{cu} \mathrm{mm}$ to $60,000 / \mathrm{cu} \mathrm{mm}$.

In view of mycosepsis with aspergillus, multiple granulomatous lesions, recurrent chest infections and unexplained hepatosplenomegaly a search for underlying immunodeficiency was carried out.

Elisa for HIV, Hepatitis B surface antigen (HbsAg) and anti-Hepatitis C serology were negative. Antinuclear factor, dsDNA were negative. T cell count was $1415 / \mathrm{cu} \mathrm{mm}$. CD4 and CD 8 ratio was normal. Immunoglobulin assay showed IgA 218 mg/dl (normal25-154 mg/dl), IgG 1900 mg/dl (960$1968 \mathrm{mg} / \mathrm{dl})$, IgM $120 \mathrm{mg} / \mathrm{dl}$ (43-196 mg/dl) and IgE $40.8 \mathrm{I} . \mathrm{U} / \mathrm{ml}$ (normal up to $50 \mathrm{I} . \mathrm{U} / \mathrm{ml})$. Nitroblue tetrazolium test (NBT) was $<20 \%$ indicating impaired phagocytic function (normal - $>90 \%$, 50$90 \%$ in carriers). NBT of the mother revealed a carrier state. Di-hydro rhodamine (DHR) test was done and stimulation index of patient was found 1.13 in respect to that of control 4.51. This test corroborated with positive NBT. C3, C4, and CH50 were within normal range. Sweat chloride test and CFTR gene analysis for $\Delta 508$ deletion were negative.

We revised our diagnosis as chronic granulomatous disease (CGD) of probable X-linked inheritance with a secondary thrombocytopenia. Prophylactic cotrimoxazole and fluconazole were started. One year of follow up revealed consistent lower values of platelet in the range of $40,000 / \mathrm{cu} \mathrm{mm}$ to $50,000 / \mathrm{cu}$ $\mathrm{mm}$. We stamped it as chronic immune thrombocytopenia.

\section{Discussion}

CGD is an inherited primary immunodeficiency disorder of phagocytic cells characterized by inability to kill catalase-positive microorganisms causing recurrent life threatening granulomatous infections ${ }^{5}$. Autoimmune disorders such as ITP, systemic lupus erythematosus, rheumatoid arthritis, sarcoidosis are reported in association with $\mathrm{CGD}^{2-4,6-9}$.

He was not suspected of having CGD at the time of first presentation with ITP. Readmission with second episode of purpura and neurological symptoms prompted further investigations which unmasked the underlying primary disorder. The normal immunoglobulin levels excluded CVID, selective IgA deficiency and Wiskott-Aldrich syndrome. Other secondary causes like HIV, Hepatitis B and Hepatitis $\mathrm{C}$, cystic fibrosis were excluded. Carrier state of the mother might further suggest this to be an $\mathrm{X}$ linked disorder.

ITP as the presenting feature of CVID has been reported $^{10}$. However; ITP in CGD is rare and even rarer as a presenting feature. Several cross-reactive auto-antibodies, produced during inefficient phagocytosis might lead to secondary autoimmune disorders ${ }^{1,3}$. These secondary ITPs are mostly 'difficult to treat ITP'. Refractory ITP in patients with CGD requiring anti-CD20 and other immunosuppressives like cyclosporine A have been reported earlier ${ }^{3,4}$.

Index case showed spontaneous improvement and a consistently stable count of $40,000 / \mathrm{cu} \mathrm{mm}$ after one year follow up without corticosteroids or any other immunosuppressives. He was stamped as 'chronic ITP'. The relatively stable course of ITP which responded to conservative management only is unique. A search for underlying primary immunodeficiency is warranted whenever a child presents with ITP on a backdrop off recurrent sinopulmonary infections.

\section{References}

1. Douglas B. C, James B. B, Howard A. L, Eline T. The ITP syndrome: pathogenic and clinical diversity. Blood. 2009; 26:6511-21.

2. Matsura R, Kagosaki Y, Tanaka Y, Kashiya H, Sanoka T, Kobayashi Y et al. A female case of chronic granulomatous disease (CGD) associated with ITP. Hiroshima Journal of Medical Science. 1980; 29:83-6.

3. Shamsian BS, Mansouri D, Pourpak Z, Rezaei N, Chavoshzadeh Z, Jadali F et al. Autosomal recessive chronic granulomatous disease, IgA deficiency and refractory autoimmune thrombocytopenia responding to Anti-CD20 monoclonal antibody. Iran Journal of Allergy, Asthma and Immunology 2008; 7:181-4.

4. Trelinski J, Chojnowski K, Kurenko-Deptuch M, Kasznicki M, Bernatowska E, Robak T. Successful treatment of refractory auto-immune thrombocytopenia with rithuximab and cyclosporine $\mathrm{A}$ in a patient with chronic 
granulomatous disease. Annals of Hematology 2005; 84: 835-6.

5. Segal BH, Leto TL, Gallin JI, Malech HL, Holland SM. Genetic, biochemical and clinical features of chronic granulomatous disease. Medicine (Baltimore) 2000; 79:170-200.

6. De Ravin SS, Naumann N, Cowen EW, Friend J, Hilligoss $\mathrm{D}$, Marquesen $\mathrm{M}$ et al. Chronic granulomatous disease as a risk factor for autoimmune disease. Journal of Allergy and Clinical Immunology 2008; 122:1097-103.

7. Schmitt CP, Scharer K, Waldherr R, Seger RA, Debatin KM. Glomerulonephritis associated with chronic granulomatous disease and systemic lupus erythematosus. Nephrology Dialysis Transplantation. 1995; 10:891-5.
8. Lee BW, Yap HK. Polyarthritis resembling juvenile rheumatoid arthritis in a girl with chronic granulomatous disease. Arthritis and Rheumatism 1994; 37:773-6.

9. De Ravin SS, Naumann N, Robinson MR, Barron KS, Kleiner DE, Ulrick J, et al. Sarcoidosis in chronic granulomatous disease. Pediatrics 2006; 117: 590-5.

10. Michel $\mathrm{M}$, Chanet $\mathrm{V}$, Galicier $\mathrm{L}$ et al. Autoimmune thrombocytopenic purpura and common variable immunodeficiency: analysis of 21 cases and review of the literature. Medicine (Baltimore) 2004; 83:254-63. 